\title{
Common Peroneal Nerve Palsy Due to Deep Tendinous Fascia Superficial to the Soleus Muscle: A Case Report
}

\author{
Corresponding author: Byung-chul Son \\ Department of Neurosurgery, Seoul St. \\ Mary's Hospital, Catholic Neuroscience \\ Institute, College of Medicine, The \\ Catholic University of Korea, 222 \\ Banpo-daero, Seocho-gu, Seoul 06591 \\ Republic of Korea \\ Tel: $+82-2-2258-6122$ \\ Fax: +82-2-594-4248 \\ E-mail: sbc@catholic.ac.kr
}

Jaeyeon Kim, Hak-cheol Koㄹ, Byung-chul Son ${ }^{1,2}$

${ }^{1}$ Department of Neurosurgery, Seoul St. Mary's Hospital, College of Medicine, The Catholic University of Korea, Seoul, ${ }^{2}$ Catholic Neuroscience Institute, College of Medicine, The Catholic University of Korea, Seoul, Republic of Korea

Received: February 28, 2018

Revised: March 24, 2018

Accepted: March 26, 2018
The posterior deep tendinous fascia of the peroneus longus muscle is known as the primary entrapment point in primary compression neuropathy of the common peroneal nerve (CPN) around the head of the fibular. However, complete decompression requires devision of all surrounding tendinous structures encroaching the nerve. We report a case of CPN palsy due to compression by deep tendinous fascia superficial to the soleus muscle. Magnetic resonance imaging was not diagnostic. The compression of the CPN by the deep tendinous fascia superficial to the soleus muscle was identified during the operation. Understanding these anatomical variants is essential to the surgeons attempting surgical decompression of the CPN at the head of the fibular, so that all compressing structures may be released appropriately.

Key Words: Decompression; Muscle, skeletal; Peroneal nerve; Peroneal neuropathies

\section{INTRODUCTION}

The common peroneal nerve (CPN) is the most commonly injured peripheral nerve of the lower extremity ${ }^{7}$. It is particularly susceptible to injury due to its superficial location, as it lies subcutaneously over the fibular neck ${ }^{2)}$. Furthermore, CPN is under tension the knee is bent.

In the entrapment neuropathy of $\mathrm{CPN}$, two constriction points along the course of CPN have been addressed. The most common site is the tendinous leading edge of the peroneus longus and posterior crural intermuscular septum ${ }^{2}$. The next potential site of entrapment corresponds to the fascial septum between the peroneal and tibial anterior muscles known as the anterior crural intermuscular septum ${ }^{2)}$. Although thick and fibrous posterior edge of the peroneus longus, under which the CPN passes, has been stressed as most common constriction point in the entrapment of CPN, compression of CPN by deep tendi- nous fascia superficial to the soleus muscle has rarely been reported.

\section{CASE REPORT}

A 38-year-old, right-handed female patient presented with a left-sided foot drop of 1 month duration. She felt paresthesia and mild cramping pain in the anterolateral aspect of left leg and dorsum of the left foot for several days. Then gradual progression of left-sided foot drop developed completely within 2 days. She was diagnosed as a foot drop and took physical therapy for one month. However, the left-sided foot drop did not improve at all and she was referred for further treatment. Her medical and family histories were unremarkable, including diabetes or hypothyroidism. She was a housewife and denied any physical trauma. Although she took regular exercise at the gymnastic, there was no specific event related to prolonged squatting position, or hard training to cause the foot drop.

On examination, weakness of ankle dorsiflexion and eversion of the left foot (NMT grade I) was noted. Although dorsiflexion of the hallux was evident, no weakness was found in the other toes. There was no abnormal finding in the sensory examination of the left foot. No Tinel sign was elicited along the course of the CPN. No tenderness or swelling was observed in her left leg and foot. Her gait was impaired for left foot drop, especially in going up the stairs. No abnormal finding was found in the x-ray examination. Magnetic resonance imaging (MRI) of the left knee was requested for investigation of unknown origin of common peroneal neuropathy. The MRI revealed high signal intensity of the muscles in the anterior compartment of the leg indicating subacute denervation (Fig. 1A). There was no abnormal high signal intensity in the CPN and no mass lesion around its course. However, the lateral edge of the soleus muscle was closely abutted to the CPN just above the fibular head (Fig. 1A). Under impression of entrapment of the left common peroneal neuropathy, exploratory decompre- 
ssion was planned.

An oblique incision was made inferior to the fibular head along the course of the CPN. We followed the technical suggestion of compression of the $\mathrm{CPN}^{2)}$. Dissection was then carried down to the underlying fascial place from the biceps tendon and iliotibial tract. The CPN was identified at the posterior edge of the peroneus longus (Fig. 1B). The superficial fascia of the peroneus longus was cut and the underlying peroneus longus muscle was retracted, revealing the leading edge of the posterior fascia. It was devided (Fig. 1B). However, tension of the posterior fascia was not significant. Rather, a trough in the underlying superficial fascia over the soleus muscle was tented to the inferior aspect of the CPN and carefully devided (Fig. 1C, D). After devision of the deep tendinous fascia superficial to the soleus muscle, the CPN lay flat smoothly around the lateral knee. The distal course of the CPN was decompressed with division of the posterior and anterior crural intermuscular septa.

The postoperative course was uneventful. There was no improvement in the motor weakness of ankle dorsiflexion and eversion of the left foot until 2-month follow-up. At the 3-month follow-up, the patient reported a complete recovery of foot drop. There was no abnormality in the sensory examination.
No residual weakness or recurrence of weakness in the dorsiflexion of left foot was found in the last follow-up at 6 postoperative months.

\section{DISCUSSION}

\section{Entrapment of the CPN}

Peroneal nerve palsy, the most common peripheral neuropathy of the lower extremity, has multiple causes. Although an extrinsic compression remains the most common cause, traumatic injuries, direct blunt trauma, metabolic diseases such as diabetes mellitus and compressive mass lesions (both intraneural and extraneural lesions) may manifest with acute or progressive peroneal nerve neuropathy ${ }^{8)}$. Recent history of significant weight loss, habitual leg crossing, and prolonged squatting are known to be associated with increased risk of peroneal palsy and acute foot drop ${ }^{8)}$. The superficial location of the $\mathrm{CPN}$, as it lies subcutaneously over the fibular neck, and its fixed position upon the hard surface of the fibular make it particular susceptible to the direct trauma or laceration ${ }^{2)}$. Nontraumatic causes include masses such as intraneural ganglion
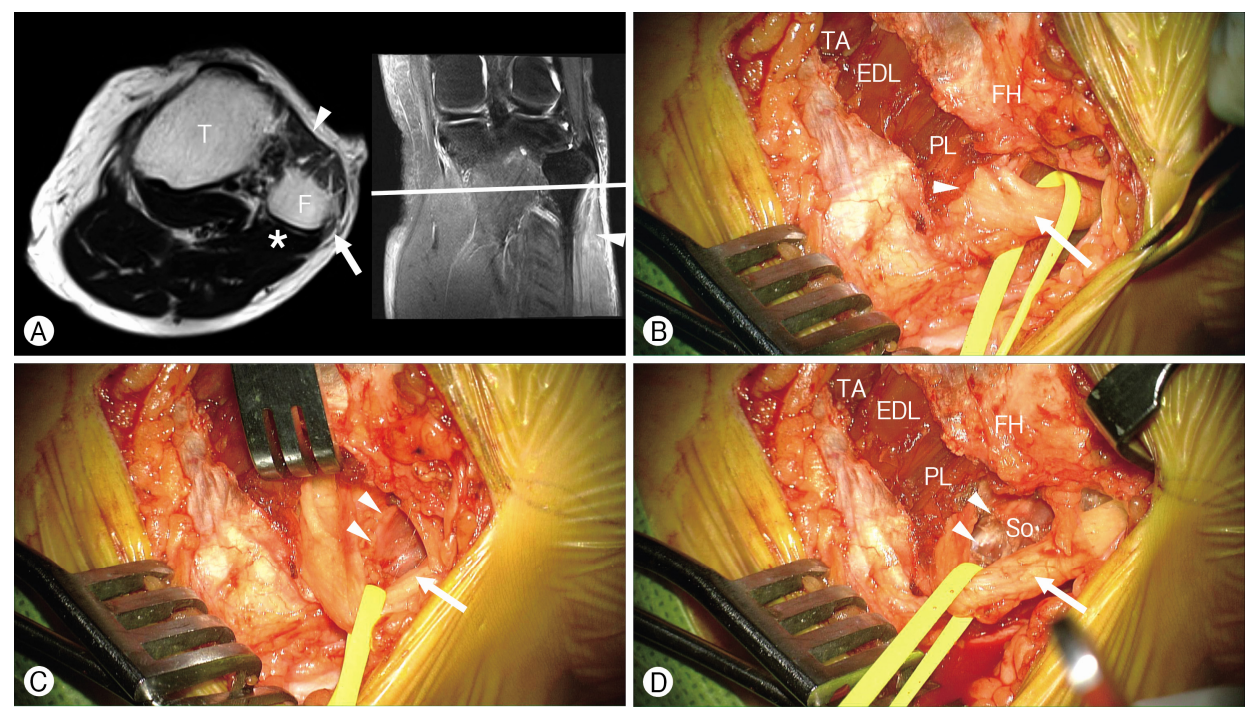

Fig. 1. Finding of common peroneal neuropathy due to deep tendinous fascia superficial to the soleus (So) muscle. (A) A T2-weighted axial, fat suppression magnetic resonance imaging of the left knee (left) showing encroachment of fascia of the So (asterisk) to the common peroneal nerve (arrow) around the head of the fibular. Signal change and mild atrophy of the muscles in the anterior compartment (arrowhead) indicating subacute denervation are evident. (B) An intra-operative photograph showing the common peroneal nerve (arrow) after division of deep tendinous fascia (arrowhead) of the peroneus longus and intermuscular septa over extensor digitorum longus and tibialis anterior muscles. (C) An intra-operative photograph showing prominent and tense deep tendinous fascia (arrowheads) superficial to the So muscle under the common peroneal nerve (arrow). (D) An intra-operative photograph showing complete decompression of the common peroneal nerve (arrow) with division of deep tendinous fascia (arrowheads) superficial to the So muscle. T: tibia; F: fibular; TA: tibialis anterior; EDL: extensor digitorum longus; $\mathrm{FH}$ : head of the fibular; $\mathrm{PL}$ : peroneus longus. 
cyst arising from the tibiofibular joint ${ }^{5,9)}$ or even rare lesions, such as nerve sheath tumors, lipomas, and osteomas ${ }^{2)}$.

\section{Anatomical Structures Related to Decompression of the CPN at the Fibular Head}

Compression of the CPN at the fibular head has been described as a compression the tunnel created by the two heads of the peroneus longus muscle ${ }^{3,7)}$. Anatomical structures that should be identified during decompression of the CPN at the fibular head include: fibrous band deep to the superficial head of the peroneus longus, fibrous septa or band on the surface of the deep head of the peroneus longus, and confluence of the origins of the soleus muscle and the peroneus longus ${ }^{3)}$. Among these, the posterior tendinous lead edge of the peroneus longus, also known as the posterior crural intermuscular septum, to be the main entrapment point of the $\mathrm{CPN}^{2}$.

According to a study regarding anatomical variations related to decompression of the CPN at the fibular head, the posterior leading edge of the peroneus longus was the most common anatomical variants found during decompression of the $\mathrm{CPN}^{3}$. The next is the fibrous septa or band on the surface of the deep head of the peroneus longus and its was found in $20 \%$. The incidence of confluence of the origin of the soleus and peroneus muscles, which is deep tendinous fascia superficial to the soleus muscle, was only $6 \%$. In the current case, compression by deep tendinous fascia superficial to the soleus muscle was suspicious in the preoperative MRI examination (Fig. 1). However, it was difficult to determine the cause of peroneal neuropathy only with vague MRI findings. Significant impingement to the posterior surface of the CPN could only identified intraoperatively (Fig. 2). Therefore, the knowledge of these anatomical variants is essential to the surgeons attempting surgical decompression of the CPN at the head of the fibular, so that all compressing structures may be released appropriately.

The results of decompression of entrapment neuropathy of the CPN are good ${ }^{4,6)}$. However, the outcome of the surgery is dependent on the etiology of the nerve injury ${ }^{1,2)}$. Surgical outcome of crush injury and gunshot wounds was worse than that of sharp injuries and knee dislocations. Although neurolysis in addition to decompression of the CPN, following knee dislocation, was recommended ${ }^{1)}$, it is not advised in primary compression neuropathy such as the current case ${ }^{2)}$.

\section{CONCLUSION}

To achieve complete decompression of the CPN, all three tendinous fibrous structures including deep fascia superficial to the soleus muscle should be identified and devided. The surgeon should aware of anatomical variants related to compression of the CPN around the head of the fibular.

\section{CONFLICTS OF INTEREST}

No potential conflict of interest relevant to this article was reported.

\section{REFERENCES}

1. Bonnevialle P, Dubrana F, Galau B, Lustig S, Barbier O, Neyret $\mathrm{P}$, et al.: Common peroneal nerve palsy complicating knee dislocation and bicruciate ligaments tears. Orthop Traumatol Surg Res 96:64-69, 2010

2. Boyd KU, Brown JM. Injury and compression neuropathy in the lower extremity. In: Mackinnon SE, Yee A eds. Nerve surgery. New York, NY: Thieme, 2015, pp338-390

3. Dellon AL, Ebmer J, Swier P: Anatomic variations related to decompression of the common peroneal nerve at the fibular head. Ann Plast Surg 48:30-34, 2002

4. Humphreys DB, Novak CB, Mackinnon SE: Patient outcome after common peroneal nerve decompression. J Neurosurg 107: 314-318, 2007

5. Kim D, Choi JG, Son BC: Peroneal nerve palsy due to subparaneurial ganglion cyst, a rare variant of intraneural ganglion cyst. Asian J Neurosurg [epub ahead of 2017.]

6. Kim DH, Murovic JA, Tiel RL, Kline DG: Management and outcomes in 318 operative common peroneal nerve lesions at the Louisiana State University Health Sciences Center. Neurosurgery 54:1421-1428, 2004

7. Mackinnon SE, Dellon AL: Surgery of the peripheral nerve. New York, NY: Thieme, 1988

8. Masakado Y, Kawakami M, Suzuki K, Abe L, Ota T, Kimura A: Clinical neurophysiology in the diagnosis of peroneal nerve palsy. Keio J Med 57:84-89, 2008

9. Spinner RJ, Atkinson JL, Tiel RL: Peroneal intraneural ganglia: the importance of the articular branch. A unifying theory. J Neurosurg 99:330-343, 2003 